\title{
Unrestricted Weight Bearing as a Method for Assessment of Nociceptive Behavior in a Model of Tibiofemoral Osteoarthritis in Rats
}

\author{
Lise Skøtt Gregersen ${ }^{1}$, Turid Røsland ${ }^{1}$, Lars Arendt-Nielsen ${ }^{1}$, \\ Garth Whiteside ${ }^{2}$, Michele Hummel ${ }^{2}$ \\ ${ }^{1}$ Center for Sensory-Motor Interaction, Aalborg University, Aalborg Ø, Denmark \\ ${ }^{2}$ Purdue Pharma LP, Cranbury, USA \\ Email: liseskoett@me.com, turid.roesland@gmail.com, lan@hst.aau.dk, \\ garth.whiteside@pharma.com,michele.hummel@pharma.com
}

Received March 20, 2013; revised April 25, 2013; accepted May 6, 2013

Copyright (C) 2013 Lise Skøtt Gregersen et al. This is an open access article distributed under the Creative Commons Attribution License, which permits unrestricted use, distribution, and reproduction in any medium, provided the original work is properly cited.

\begin{abstract}
Background: Novel preclinical models for prediction of osteoarthritis-like pain are necessary for the elucidation of osteoarthritis (OA) pathology and for assessment of novel analgesics. A widely used behavioral test in rat models of tibiofemoral OA is hind limb weight bearing (WB). However, this method evaluates WB in an unnaturally restricted manner. The aim of this study was therefore to characterize the Tekscan Pressure Measurement System as a means to assess OA-like tibiofemoral pain in rats by determination of plantar pressure distribution in a more natural and unrestricted position, defined as unrestricted WB. Methods: Intra-articular injections of $1 \mathrm{mg}$ monosodium iodoacetate (MIA) or saline were administrated in the left hind knee of 84 male Sprague Dawley rats. Changes in unrestricted WB between ipsilateral and contralateral hindlimbs were determined. Morphine ( $5 \mathrm{mg} / \mathrm{kg}$ administered subcutaneously) and naproxen (60 mg/kg per-oral) were examined for their ability to reverse WB changes. Results: Changes in hind limb unrestricted WB were observed $14(P<0.05), 21(P<0.001)$ and $28(P<0.001)$ days post intra-articular injections of MIA compared to control. These alterations were attenuated by morphine 1 hour post administration compared to baseline but were not affected by naproxen. Conclusion: This study indicated that unrestricted WB assessed by the Tekscan system can be utilized as a clinically relevant method to assess aberrations in WB induced by intra-articular MIA injections in rodents. Therefore, this system may be useful for understanding the mechanisms of OA pain in humans and may also assist in the discovery of novel pharmacological agents.
\end{abstract}

Keywords: Osteoarthritis; Pain; Monosodium Iodoacetate; Unrestricted Weight Bearing; Morphine; Naproxen

\section{Introduction}

Osteoarthritis (OA) is an age-related disease affecting millions of people worldwide [1,2]. OA symptoms vary considerably and patterns of nociception are distinguished differently by patients varying from dull aches to sharp stabbing pain. Neuropathic descriptors apply to a subset of OA patients indicating a role of sensitization in the underlying pathology [3].

$\mathrm{OA}$ is primarily characterized by damaged articular cartilage and subsequent cartilage loss [1] though novel research confirms that several joint tissues are affected $[4,5]$. The joint changes induce functional limitations, primarily due to pain $[1,4]$. Continuous nociceptive input from an OA damaged knee in animals drive central sensitization [6], which has recently been shown in knee OA patients [7]. However, the complexities of the pathology of OA nociception remain unclear for which reason pain relief is insufficient [1,8]. Therefore, novel preclinical models and endpoints that are more predictive of the human condition are necessary for a better physiological understanding of OA pain and for the development and optimization of efficacious therapies.

Intra-articular (i.a.) administration of monosodium iodoacetate (MIA) in rats is a validated chemical model used to investigate OA. MIA, when injected through the patellar tendon in the hind limb of rodents, displays multiple components of disease progression and symptoms similar to human OA and induces significant nociceptive behavior [9-11] albeit with a different time course [12]. Alterations in hind limb weight bearing (WB) measured 
by an incapacitance test are widely used as a nociceptive behavioral outcome in the MIA model as the rat will carry less weight on a painful limb. This model evaluates static, resting nociceptive behavior by assessing WB alterations in a confined, immobilized position where the majority of the body weight is directed over the hind limbs [9,11], here defined as restricted WB. However, assessment of load bearing in an unrestricted and more natural position on all four limbs would be preferable.

The Tekscan Very HR Walkway Floor Mat Pressure Measurement System (Tekscan Inc., USA) is available for the study of plantar pressure in animals by analysis of the force applied to the ground per limb over time. This method seems clinically relevant as it has potential to evaluate WB while the rats are unrestricted in a freely moving position. Moreover, the methodology offers some pre-clinical translatability as the Tekscan device is already used for WB assessment in humans.

This study aimed to characterize the Tekscan system as a means to assess tibiofemoral OA-related nociception by determination of plantar pressure distribution in MIA injected rats in an unrestricted position, defining the method as unrestricted WB. For further validation of unrestricted WB as a relevant, non-evoked behavioral measure or endpoint for pain the effects of two clinically available analgesics on behavior were examined.

\section{Material and Methods}

\subsection{Animals}

All experiments were approved by the Institutional Animal Care and Use Committee at Purdue Pharma L.P. and were in strict accordance with the ethical guidelines laid down by the International Association for the Study of Pain [13] for the care and use of laboratory animals. 84 male Sprague Dawley rats (150 - 200 grams, Harlan Labs, USA) were included in the study. Rats were grouphoused in temperature and humidity controlled rooms on a 12-h light/dark cycle with access to food and water ad libitum, except before oral (p.o.) administration of drugs when food was removed for 16 - 18 hours prior to dosing.

\subsection{Induction of Tibiofemoral Osteoarthritis}

For the induction of the unilateral tibiofemoral OA model, 84 animals were briefly anesthetized with isoflurane 2.5\% - 4.0\% (Baxter Healthcare, USA). Their left hindlimb was shaved and sanitized with Betadine Surgical Scrub (Purdue Pharma, LP, USA) and 72\% ethanol. Using a 27-gauge needle through the patellar tendon in the left hind limb, 42 rats received a single i.a. injection of 1 mg MIA (Sigma-Aldrich, USA) (1 mg in $0.02 \mathrm{ml}$ normal saline), while 42 rats (control group) received a single i.a. injection of $0.02 \mathrm{ml}$ normal saline. The rats were allowed to fully recover in a temperature-controlled cage and then returned to their home cages. The dose of MIA used (1 $\mathrm{mg} /$ i.a. injection) was selected based on in-house efficacy data as well as from the literature $[9,11]$.

\subsection{Pain Behavioral Testing}

Assessment of bilateral unrestricted WB following unilateral injection of MIA was used as behavioral measurement of nociception in OA-induced rats. Restricted WB was also assessed and used as a reference value. Rats were tested at day 0 (baseline), 7, 14, 21 and 28 postMIA injection.

\subsubsection{Unrestricted Weight Bearing}

Changes in weight distribution between the left and right hind limbs and front limbs were assessed by unrestricted WB using the Tekscan Walkway ${ }^{\mathrm{TM}}$ 5101D HR Floor Mat Pressure Measurement System (Tekscan, Inc. South Boston, MA, USA). The Tekscan system is composed of two 5101 very High-Resolution (HR) sensors with a spatial resolution of 15.5 sensels $/ \mathrm{cm}^{2}$ embedded into a floor mat. For the purpose of this experiment, the sensors were calibrated to a known weight of $268.5 \mathrm{~g}$ according to manufacturer instructions (Tekscan Walkway ${ }^{\mathrm{TM}}$ Software version 7.01, Tekscan Inc. South Boston, MA, USA). Rats were positioned on an open surface inside a $22.4 \times 11.2$ $\mathrm{cm}$ clear Plexiglas chamber, which was placed on top of two sensors without contact between sensels and chamber. In order to acclimate to the equipment and to provoke joint pain, the rats were allowed to walk freely around on the mat and when hind limbs and front limbs were in an equal position (Figure 1(a)), the downward force applied by each limb (measured in gram) was read over a six second period with a frequency of 100 frames per second. Each data point is the average of two readings. Data were computed in the Tekscan Walkway ${ }^{\mathrm{TM}}$ Software and then imported into an Excel spreadsheet. Unrestricted WB measurements were recorded before (baseline) and after the i.a. procedure (model development). Rats were randomized to treatment group following model induction.

\subsubsection{Restricted Weight Bearing}

Changes in restricted weight distribution between the left and right hind limbs were determined using incapacitance testers (Columbus Instruments, USA). Rats were placed in an angled Plexiglas chamber so that each hind limb was positioned on a separate force plate (Figure 1(b)). The rats were allowed to acclimate to the apparatus and when stationary, readings were taken. The downward force (measured in grams) applied by each hind limb was assessed and averaged over a three second period. Each data point is the average of three readings. 


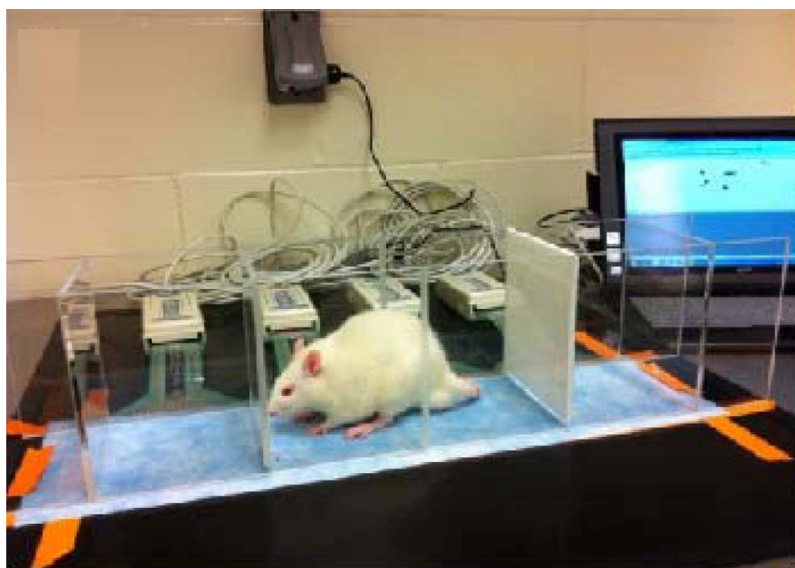

(a)

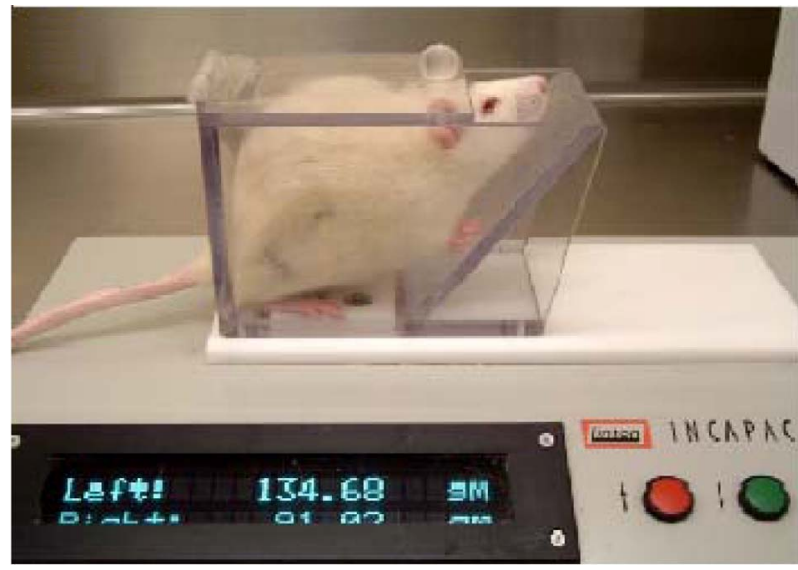

(b)

Figure 1. Weight bearing assessment: (a) Assessment of unrestricted weight bearing by the Tekscan system. (b) Assessment of restricted weight bearing by the incapacitance meter.

Similarly, restricted WB measurements were recorded before (baseline) and after the i.a. procedure (model development). Rats were randomized to treatment group following model induction.

\subsection{Drugs, Vehicles, and Dosing}

Naproxen sodium (Sigma-Aldrich, USA) was dissolved in $0.5 \%$ methylcellulose and administered p.o. at a dose of $60 \mathrm{mg} / \mathrm{kg}$ while morphine sulfate (Sigma-Aldrich, USA) was dissolved in $0.9 \%$ normal saline and administered at a dose of $5 \mathrm{mg} / \mathrm{kg}$ subcutaneously (s.c.). The dose volumes were $5 \mathrm{ml} / \mathrm{kg}$ and $2 \mathrm{ml} / \mathrm{kg}$, respectively. Control rats were dosed similarly with the appropriate matched vehicle. The doses for both compounds were selected based on in-house assessment as well as efficacy results obtained from several nociceptive models described in the literature [9,11,14-15].

The effect of naproxen on WB was assessed using rats 14 days post-MIA/saline injection. Rats ( $n=10$ /group) were given a single dose of naproxen or vehicle. Changes in hind limb weight distribution were determined by unrestricted and restricted WB as outlined above, before compound administration (pre-drug baseline; day 14) and 1,3 , and 5 hours post-compound administration.

The effect of morphine on WB was assessed in a separate cohort of rats 21 and 28 days post-MIA/saline injection ( $n=12$ /group and $n=20$ /group, respectively). Rats were given a single dose of morphine or vehicle. Changes in limb weight distribution were determined by unrestricted and restricted WB as outlined above before compound administration (pre-drug baseline; days 21 and 28) and 1 and 3 hours post-compound administration.

\subsection{Pain Behavioral Analysis}

For unrestricted and restricted WB measurements, the percent weight (in grams) borne on the left limb was determined using the following formula:

\% weight on left limb

$=\left[\frac{\text { weight on left limb }}{\text { weight on left limb + weight on right limb }}\right] \times 100$

Data were computed in Microsoft ${ }^{\circledR}$ Office Excel $^{\circledR} 2007$ (Microsoft ${ }^{\circledR}$ Corporation, USA).

\subsection{Statistical Analysis}

The statistical analyses were carried out using GraphPad Prism (version 5.04; GraphPad Software, Inc., USA). The analyses for the time course effect on the hind limb unrestricted and restricted WB following injection of MIA versus saline controls and the effect of administration of analgesic compounds were conducted using a two-way analysis of variance (ANOVA). The analyses of the effect of treatment within groups were performed using a one-way ANOVA. Bonferroni multiple comparison test was performed as a post hoc comparison. Data are presented as mean \pm SEM. $P<0.05$ was set as the level of statistical significance.

\section{Results}

\subsection{Unrestricted Weight Bearing versus Restricted Weight Bearing}

The i.a. administration of either MIA or saline had no effect on the general health of the animals. The animals in the MIA- and saline-injected groups gained body weight normally and neither displayed any signs of distress or infection. Following MIA administration a timedependent increase in hind limb joint pain was noted throughout the 28-day study period. The pain was defined by changes in hind limb weight distribution as- 
sessed by both unrestricted and restricted WB (Figures 2(a) and Figure 2(b)). When compared to the salineinjected group, the MIA-injected group exhibited a decrease in hind limb WB of the ipsilateral limb.

The MIA model resulted in a significant decrease in the mean percentage of weight distribution on the affected hind limb, $41.0 \% \pm 1.8 \%$ on day $21(P<0.001)$ and $40.0 \% \pm 2.7 \%$ on day $28(P<0.001)$ compared to a between days average of $51.1 \% \pm 1.1 \%$ for controls (Figure 2(a)) as determined by unrestricted WB. Although numerically lower, the values on days 7 and 14 were not significantly different from controls in this cohort of animals. No significant changes were found in front limb weight distribution assessed by this methodology (Figure 3) for which reason no further investigations of front limb WB were obtained.

Similarly, the MIA model resulted in a significant decrease in the mean percentage of weight on the ipsilateral hind limb, $43.6 \% \pm 0.0 \%$ at day $7(P<0.0001), 44.00 \%$ $\pm 0.0 \%$ at day $14(P<0.0001), 42.2 \% \pm 0.0 \%$ at day 21 $(P<0.0001)$, and $40.7 \% \pm 0.0 \%$ at day $28(P<0.0001)$ compared to a between days average of $50.0 \% \pm 0.0 \%$ for controls (Figure 2(b)) as determined by the restricted WB methodology.

\subsection{Pharmacological Modulation of Unrestricted Pain Behavior}

Experiments designed to determine the analgesic effects of naproxen and morphine on MIA-induced OA-like pain were evaluated by weight distribution assessment between the hind limbs. Behavior was assessed on days 14, 21 and 28 post-MIA injection. In order to ensure that the compounds used above were not impairing the WB assessment on their own, the compounds were administered to saline-injected rats as well. None of the compounds had any significant effect on WB in the control rats.

\subsubsection{Naproxen}

On day 14 naproxen did not significantly reverse the deficits in hind limb weight distribution noted between MIA- and saline-injected animals $(P<0.05)$ using unrestricted WB assessment (Figure 4(a)). However, naproxen did significantly reverse the alterations in hind limb weight distribution noted when assessed by restricted WB 1, 3 and 5 hours post-naproxen administration.

The mean percentages of weight noted on the ipsilateral hind limb were $50.3 \% \pm 0.0 \%(P<0.0001), 48.1 \% \pm$ $0.0 \%(P<0.0001)$, and $49.6 \% \pm 0.0 \%(P<0.0001)$ at each of the time points, respectively, compared to an average of $45.7 \% \pm 0.0 \%$ for saline-injected controls (Figure 4(b)).

\subsubsection{Morphine}

Morphine administration on days 21 and 28 post MIA-
Unrestricted Hind Limb Weight Bearing

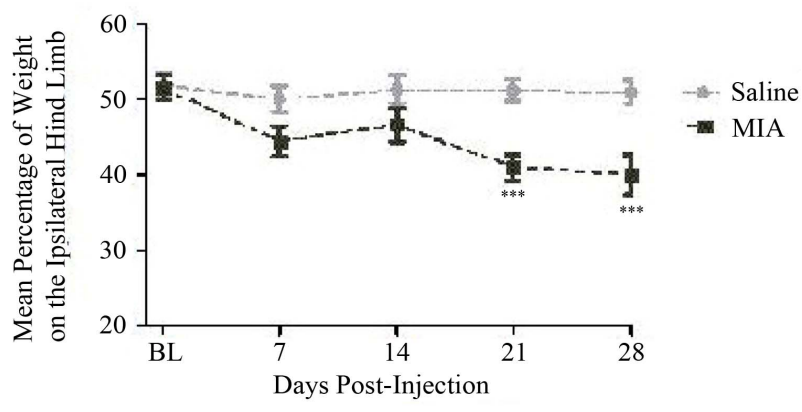

(a)

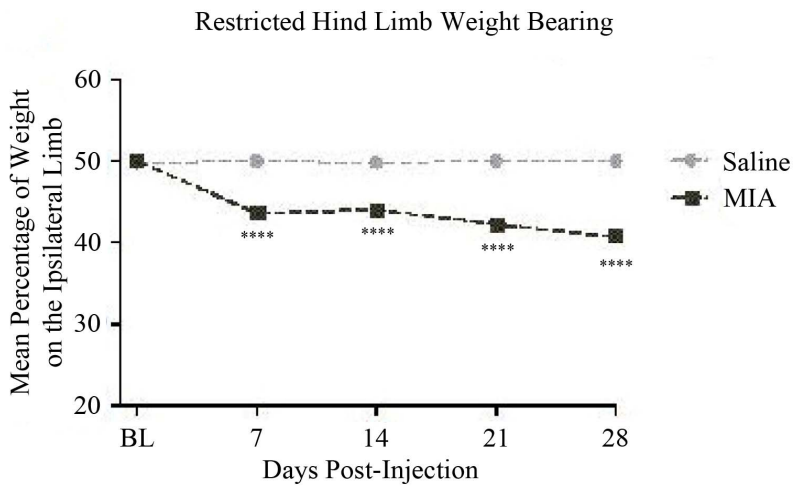

(b)

Figure 2. Unrestricted versus restricted hind limb weight bearing: (a) MIA (1 mg, 20 microliters) or saline (20 microliters) was injected intra-articularly into the left patellar tendon of Male, Sprague-Dawley rats, $150-200 \mathrm{~g}, n=$ 20/group. Unrestricted and (b) restricted weight bearing of the hind limbs were assessed on days 0 (baseline), 7, 14, 21, and 28. Data are shown as mean \pm SEM. Data were analyzed by a two-way ANOVA using a Bonferroni multiple comparison test where ${ }^{* * *} P<0.001$ and ${ }^{* * * *} P<0.0001$.

Unrestricted Front Limb Weight Bearing

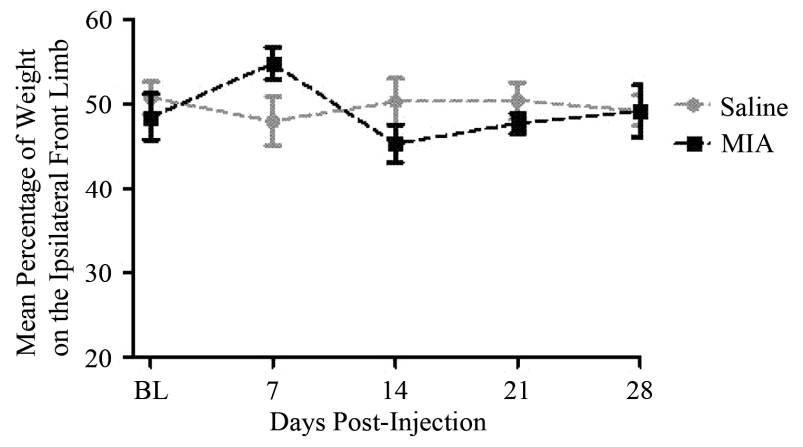

Figure 3. Unrestricted front limb weight bearing: MIA (1 mg, 20 microliters) or saline (20 microliters) was injected intra-articularly into the left patellar tendon of Male, Sprague-Dawley rats, $150-200$ g, $n=20$ group. Unrestricted weight bearing of the front limbs was assessed on days 0 (baseline), 7, 14, 21, and 28. Data are shown as mean \pm SEM. Data were analyzed by a two-way ANOVA using a Bonferroni's Multiple Comparison test. 


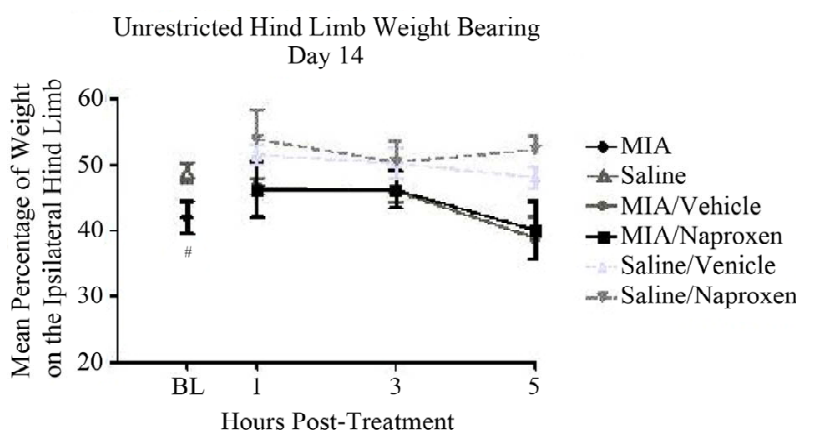

(a)

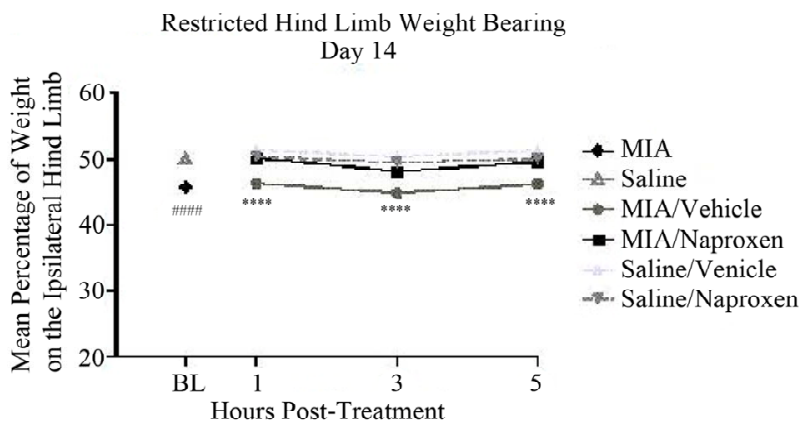

(b)

Figure 4. Unrestricted versus restricted hind limb weight bearing, day 14: (a) Naproxen sodium $(60 \mathrm{mg} / \mathrm{kg})$ or $0.5 \%$ methylcellulose (vehicle) was administered p.o. $1 \mathrm{~h}$ prior to testing. Male, Sprague-Dawley rats $(280-310$ g; $n=5$ /group) received a single intra-articular injection of either saline or MIA (1 mg/20 microliters) into the left hind limb. Unrestricted weight bearing was assessed with Tekscan Very HR Walkway Floor Mat Pressure Measurement System and (b) restricted weight bearing was assessed with incapacitance testers. Data were analyzed using a two wayANOVA followed by Bonferroni's Multiple Comparison test where ${ }^{\#} P<0.05$ and ${ }^{\# \# \#} P<0.0001$ MIA/Vehicle vs. Saline/Vehicle [model development] and ${ }^{* * * *} \boldsymbol{P}<0.0001$ MIA/ Vehicle vs. MIA/Naproxen. Data are represented as mean \pm SEM.

injection tended to normalize the changes in hind limb weight distribution assessed by unrestricted WB 1 and 3 hours post-exposure as compared to vehicle treated controls. The mean percentage of weight on the affected hind limb on day 21 increased to $49.6 \% \pm 3.6 \%$ and $47.5 \% \pm 0.7 \%$ at 1 and 3 hours post-morphine administration, respectively, compared to an average of $42.7 \% \pm$ 1.1\% for vehicle treated animals (Figure 5(a)). A similar effect was noted on day 28. On day 28, the mean percentage of weight on the affected hind limb increased to $49.7 \% \pm 3.1 \%$ and $43.4 \% \pm 3.7 \%$ at 1 and 3 hours postmorphine administration, respectively, compared to an average of $37.5 \% \pm 2.7 \%$ for vehicle treated controls (Figure 5(b)). However, none of these differences were statistically significant.

When using the restricted WB methodology, morphine significantly reversed the changes in hind limb weight

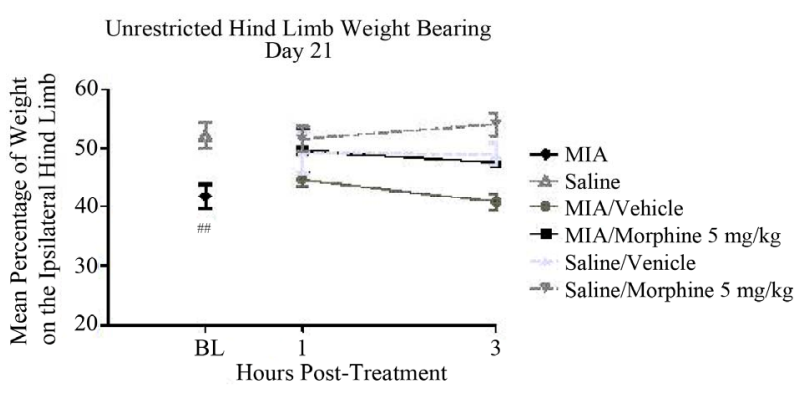

(a)

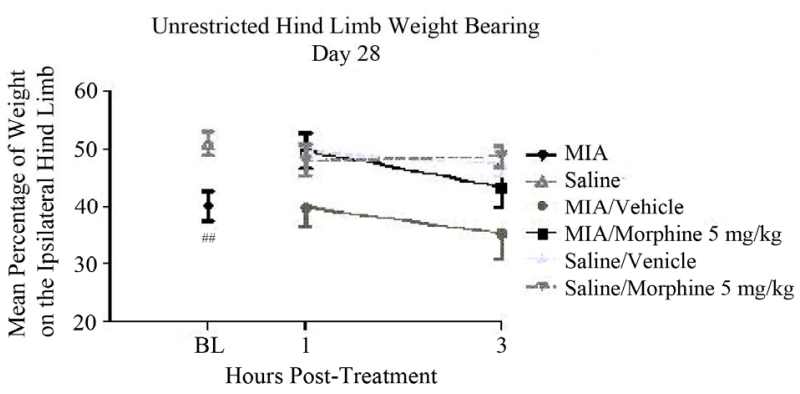

(b)

Figure 5. The effect of morphine on unrestricted Hind limb weight bearing: (a) Morphine sulfate $(5 \mathrm{mg} / \mathrm{kg}$ ) or $0.9 \%$ Saline (vehicle) were administered s.c. 1 or 3 hours prior to testing. Male, Sprague-Dawley rats $(280$ - $325 \mathrm{~g} ; n=$ 6/group) received a single intra-articular injection of either saline or MIA (1 mg/20 microliters) into the left hind limb. Unrestricted weight bearing was assessed with Tekscan Very HR Walkway Floor Mat Pressure Measurement System on days 21 and (b) 28 post-model development. Data were analyzed using a two way-ANOVA followed by Bonferroni's Multiple Comparison test where ${ }^{\# \#} P<0.01$ MIA/ Vehicle vs. Saline/Vehicle [model development]. Data are represented as mean $\pm \mathrm{SEM}$.

distribution 1 and 3 hours post treatment when compared to vehicle treated OA rats both at day 21 and day 28 post-MIA injections.

The mean percentage of weight on the ipsilateral hind limb on day 21 increased to $50.8 \% \pm 0.0 \%$ at 1 hour $(P<$ $0.0001)$ and $47.5 \% \pm 0.0 \%$ at 3 hours $(P<0.0001)$ post-morphine administration compared to an average of $44.7 \% \pm 0.0 \%$ for vehicle controls (Figure 6(a)). On day 28 the mean percentage of weight on the affected limb was increased to $49.5 \% \pm 0.0 \%$ at 1 hour $(P<0.0001)$ and $48.3 \% \pm 0.0 \%$ at 3 hours $(P<0.0001)$ post-morphine administration compared to an average of $39.3 \% \pm 0.0 \%$ for vehicle treated rats (Figure 6(b)). Morphine treatment did not affect WB in the control animals.

\section{Discussion}

The MIA model of OA-like pain produced deficits in unrestricted hind limb WB as evaluated by the Tekscan system. Morphine attenuated this WB deficit within the group of OA rats albeit with a limited effect suggesting 


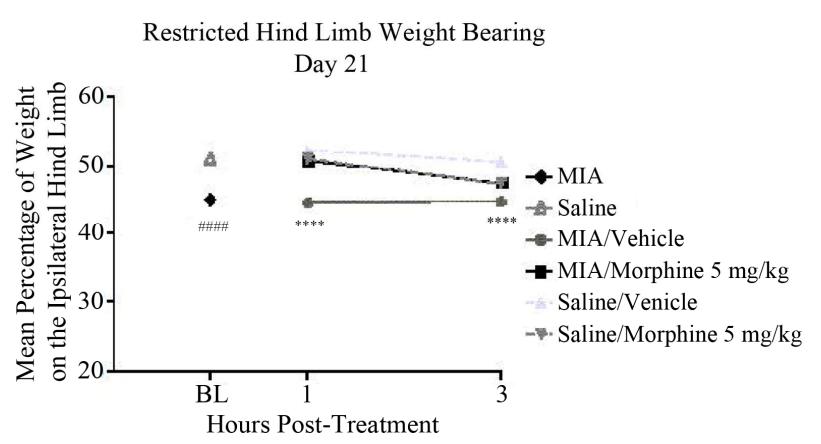

(a)

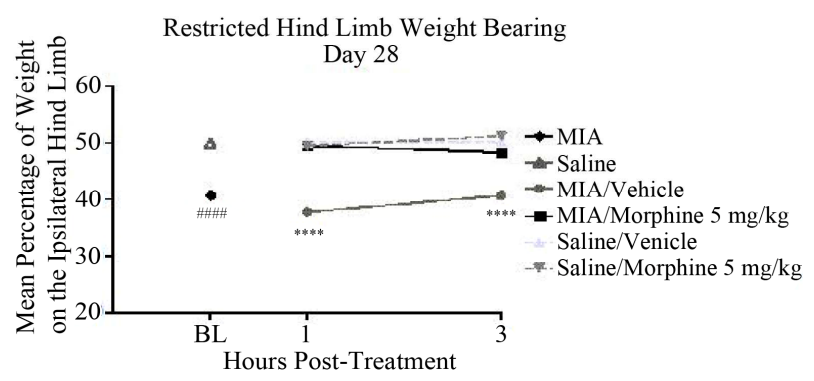

(b)

Figure 6. The effect of morphine on restricted hind limb weight bearing: (a) Morphine sulfate $5 \mathrm{mg} / \mathrm{kg}$ or $0.9 \%$ Saline (vehicle) was administered s.c. 1 or 3 hours prior to testing. Male, Sprague-Dawley rats $(280$ - $325 \mathrm{~g} ; n=$ 10 /group) received a single intra-articular injection of either saline or MIA (1 mg/20 microliters) into the left hind limb. Restricted weight bearing was assessed with incapacitance testers on days 21 and (b) 28 post-model development. Data were analyzed using a two way-ANOVA followed by Bonferroni's Multiple Comparison test where ${ }^{\# \# \# \# P<0.0001}$ MIA/Vehicle vs. Saline/Vehicle [model development] and $^{* * * *} \boldsymbol{P}<0.0001$ MIA/Vehicle vs. MIA/Morphine. Data are represented as mean \pm SEM.

that unrestricted hind limb WB can be assessed by the Tekscan system.

The lack of methods measuring primary hyperalgesia and allodynia has led to the development of several assessment techniques for OA pain measurement in rats (e.g. the validated and frequently used method of restricted hind limb WB published by Bove, et al. [9], Pomonis, et al. [11], Chu, et al. [16], and Schött, et al. [17]); all of which are an index for static or resting pain behavior as the rats are constricted during the assessments. Moreover, a limited number of studies $[10,14,16]$ have investigated movement-induced OA precipitated pain behavior in rats by assessment of hind limb compressive grip force and demonstrated relevant findings. However, limitations are associated with the grip force method. More specifically, Chandran, et al. [14] found that morphine reduces the grip force strength in naïve rats. This is concerning and problematic for all compounds producing sedative-like effects, not just when using the grip force method but also in other methods testing movement-induced pain.

\subsection{The Tekscan System as a Method of Unrestricted Weight Bearing}

The Tekscan system has previously demonstrated efficient, reliable, and accurate analysis of behavioral changes that accompany hind limb paresis in rodents during gait [18] and therefore the Tekscan system is relevant to use in investigations of OA-like pain. To our knowledge the only study using the Tekscan system for pain-induced WB deficits in OA rats was published recently by Rashid, et al. [19]. Using a WB method comparable to the unrestricted WB method described herein, the authors found a significant decrease in $\mathrm{WB}$ on the ipsilateral hind limb and a significant increase in WB on the contralateral hind limb of OA rats. Similarly, the authors demonstrated the relevance of the Tekscan system as a method for assessment of OA-like pain in rodents as confirmed in our study. An increasing number of studies [18,20-22] have investigated gait in OA rats, though Clarke, et al. [23] found that neither velocity of locomotion, stride length, nor stance, stride or swing times, were altered in OA rats when compared to controls. Load bearing of the limbs was the only parameter tested showing a significant alteration during gait [23] indicating that Tekscan system measurement of unrestricted WB during an unrestricted stance position is a relevant method.

The observed changes in unrestricted hind limb WB were only statistically significant on days 14 (pharmacology), 21 and 28 post induction of OA, whereas restricted hind limb WB demonstrated statistically significant changes on all assessment days indicating that the restricted WB may be a more sensitive pain indicator. However, while more sensitive, it may be a less relevant measure as rodents are quadrupeds. In the light of this the insignificant unrestricted WB results noted on day 7 post-induction of OA should not be considered a limitation of the Tekscan method. Previous studies $[11,14,24]$ have demonstrated that pharmacological studies using the MIA-model are most clinically relevant when performed at later time points, such as $21+$ days post-MIA injection, because of the biphasic nature of MIA injections. Therefore, in the current study the efficacy of naproxen and morphine was assessed 14 - 28 days postinjection.

Previous studies [9,11] have described measurements of hind limb weight distribution as a reliable and practical method to assess nociception associated with experimental OA. The current results are in accordance with those studies, which serve in the validation of the Tekscan system. When comparing unrestricted WB to restricted WB, the values observed were similar indicating that the two methodologies are reliable and that the ani- 
mals are not stressed. Ferreira-Gomes, et al. [20] demonstrated, by use of another method of unrestricted WB on all four limbs, a higher correlation between decreased $\mathrm{WB}$ in an OA limb and sensitivity to the normal movement of the OA knee than between decreased $\mathrm{WB}$ in an $\mathrm{OA}$ limb and secondary allodynia, thereby suggesting that decreased $\mathrm{WB}$ in the $\mathrm{OA}$ limb is a consequence of the sensitivity in the knee joint rather than secondary allodynia in the paw. Hence, we surmise that the decreased WB noted in the current study is due to an increased sensitivity to mechanical stimuli resulting from damage of the knee articulation induced by MIA.

The Tekscan system offers a comprehensive approach for measuring plantar pressure from front limbs and hind limbs and can thereby reveal possible compensatory patterns in the front limbs and contralateral hind limb as a result of unilateral OA induction in rodents. To our knowledge, only a few studies [18-19] have investigated WB of the front limbs after injury to one of the hind limbs. Boyd, et al. [18] demonstrated that rats with unilateral sciatic nerve injury had significantly increased WB on their contralateral front limb during gait when measured with the Tekscan system. Rashid, et al. [19] demonstrated that the decrease of WB on the ipsilateral hind limb in MIA rats was compensated mostly by the contralateral hind limb and only a smaller fraction of the compensated weight was borne on the front limbs. The present study did not demonstrate any difference in front limb WB post MIA-induced tibiofemoral OA. Interestingly, this study found that the rat only bears $29.5 \% \pm$ $0.7 \%$ (data not shown) of its total load on the front limbs. Given this, we have concluded that the front limbs only partially contribute to the model of unrestricted WB compared to the hind limbs. However, standing on all four limbs seemed to demonstrate a more natural adaptation to the environment and the nociceptive situation compared to the method of restricted WB. This, however, is in contrast to a study published by Tétreault, et al. [22]. The investigators observed that several of the rats were liable to pose only supported by the contralateral front limb though, looking like their preferred position. However, movies were only taken when all four limbs had contact with the sensors and therefore the movies might not picture the preferred position of the rat. Hence the criteria for a correct position should be reconsidered in further studies as the position of the front limbs might influence the load on the hind limbs.

\subsection{Pharmacological Assessment by Unrestricted Weight Bearing}

\subsubsection{Naproxen}

Non-steroidal anti-inflammatory drugs (NSAIDs) and selective cyclooxygenase 2 inhibitors (COX-2) are some of the most frequently prescribed drugs for the management of OA pain [25]. Therefore, the effect of naproxen sodium was investigated on MIA-induced changes in WB. On day 14 post-MIA injection, naproxen sodium did not attenuate any of the deficits noted with either the unrestricted or restricted WB endpoints. This is partly in line with a previous study conducted by Pomonis, et al. [11] who demonstrated that acute administration of indomethacin or celecoxib do not reduce MIA-induced alterations in restricted WB. The authors found that chronic administration is necessary to produce a partial restoration of the restricted WB deficit noted [11]. Additionally, Rashid, et al. [19] found that only the first week post-MIA injection was sensitive to the effects of naproxen on unrestricted WB whereas the third week post-MIA injection was without effect. Although these results are consistent with findings in the clinic [26], additional chronic treatment paradigms are necessary to ascertain the overall efficacy of NSAIDs and COX-2 inhibitors on the unrestricted WB endpoint and to gain insight on any inherent translational value.

\subsubsection{Morphine}

Morphine is widely used in animal models investigating OA-related joint pain [11,14-15,27]. The maximum antinociceptive effect of morphine sulfate has been determined to occur between 30 and 90 minutes when dosed at $6 \mathrm{mg} / \mathrm{kg}$ [27]. In the current study, morphine significantly reversed the unrestricted hind limb WB deficit 1 hour post-administration compared to vehicle-injected OA rats $(P<0.05)$. The efficacy of morphine in this OA model is in accordance with previous studies $[11,15,27]$ where similar doses of morphine had anti-nociceptive effects demonstrated by WB changes. In a human study, morphine was efficacious in reducing pain in patients with moderate-to-severe OA [28]. The demonstrated antinociceptive effect of morphine provides support for the endpoint of unrestricted $\mathrm{WB}$ as a reliable method to evaluate MIA-induced pain.

\section{Conclusion}

Pain associated with OA still continues to represent a major unmet medical need. Therefore, novel and effective pharmacological therapeutic options to treat OA pain are still very much warranted. Animal models of OA like MIA continue to be used to provide new information in our evaluation of nociception parameters relevant to the human condition. In this regard, the present study indicates that the Tekscan system can be utilized to assess OA-like pain behavior in rats using a clinically relevant endpoint, unrestricted WB. Although additional pharmacological validation studies are needed to ultimately determine the utility of the Tekscan system as a transla- 
tional tool, initial results from this study and others are promising [19,22,29].

\section{REFERENCES}

[1] J. W. J. Bijlsma, F. Berenbaum and F. P. J. G. Lafeber, "Osteoarthritis: An Update with Relevance for Clinical Practice,” Lancet, Vol. 377, No. 9783, 2011, pp. 21152126. doi:10.1016/S0140-6736(11)60243-2

[2] H. Bliddal, L. Bilde, A. Ankjær-Jensen, T. Demontis, K. E. Jensen, H. Lund, A. Odgaard, J. O. Rasmussen, H. Røgind, H. Schrøder and K. L. Sørensen, "Referenceprogram for Behandling af Knæartrose,” 2007. http://www.sst.dk/publ/Publ2007/PLAN/SfR/Refprg_kna eartrose.pdf

[3] J. R. Hochman, M. R. French, S. L. Bermingham and G. A. Hawker, "The Nerve of Osteoarthritis Pain," Arthritis Care \& Research, Vol. 62, No. 7, 2010, pp. 1019-1023. doi:10.1002/acr.20142

[4] D. T. Felson, "Developments in the Clinical Understanding of Osteoarthritis,” Arthritis Research \& Therapy, Vol. 11, No. 1, 2009, pp. 203-214. doi:10.1186/ar2531

[5] M. B. Goldring and S. R. Goldring, “Articular Cartilage and Subchondral Bone in the Pathogenesis of Osteoarthritis," Annals of the New York Academy of Sciences, Vol. 1192, No. 1, 2010, pp. 230-237. doi:10.1111/j.1749-6632.2009.05240.x

[6] H. G. Schiable, "Spinal Mechanisms Contributing to Joint Pain,” Novartis Foundation Symposia, Vol. 260, 2004, pp. 4-22. doi:10.1002/0470867639.ch2

[7] L. Arendt-Nielsen, H. Nie, M. B. Laursen, B. S. Laursen, P. Madeleine, O. H. Simonsen and T. Graven-Nielsen, "Sensitization in Patients with Painful Knee Osteoarthritis,” Pain, Vol. 149, No. 3, 2010, pp. 573-581. doi:10.1016/j.pain.2010.04.003

[8] J. S. Lawrence, J. M. Bremner and F. Bier, “Osteoarthrosis. Prevalence in the Population and Relationship between Symptoms and X-Ray Changes," Annals of the Rheumatic Diseases, Vol. 25, No. 1, 1966, pp. 1-24.

[9] S. E. Bove, S. L. Calcaterra, R. M. Brooker, C. M. Huber, R. E. Guzman, P. L. Juneau, D. J. Schrier and K. S. Kilgore, "Weight Bearing as a Measure of Disease Progression and Efficacy of Anti-Inflammatory Compounds in a Model of Monosodium Iodoacetate-Induced Osteoarthritis," Osteoarthritis Cartilage, Vol. 11, No. 11, 2003, pp. 821-830. doi:10.1016/S1063-4584(03)00163-8

[10] Y. Lee, M. Pai, J. D. Brederson, D. Wilcox, G. Hsieh, M. F. Jarvis and R. S. Bitner, "Monosodium IodoacetateInduced Joint Pain Is Associated with Increased Phosphorylation of Mitogen Activated Protein Kinases in the Rat Spinal Cord,” Molecular Pain, Vol. 7, 2011, pp. 3949. doi:10.1186/1744-8069-7-39

[11] J. D. Pomonis, J. M. Boulet, S. L. Gottshall, S. Phillips, R. Sellers, T. Bunton and K. Walker, "Development and Pharmacological Characterization of a Rat Model of Osteoarthritis Pain,” Pain, Vol. 114, No. 3, 2005, pp. 339346. doi:10.1016/j.pain.2004.11.008

[12] S. Orita, T. Ishikawa, M. Miyagi, N. Ochial, G. Inoue, Y.
Eguchi, H. Kamoda, G. Arai, T. Toyone, Y. Aoki, T. Kobu, K. Takekazu and S. Ohtori, "Pain-Related Sensory Innervation in Monoiodoacetate-Induced Osteoarthritis in Rat Knee That Gradually Develops Neuronal Injury in Addition to Inflammatory Pain,” BMC Musculoskeletal Disorders, Vol. 12, 2011, pp. 134-146. doi:10.1186/1471-2474-12-134

[13] M. Zimmermann, "Ethical Guidelines for Investigations of Experimental Pain in Conscious Animals,” Pain, Vol. 16, No. 2, 1983, pp. 109-110. doi:10.1016/0304-3959(83)90201-4

[14] P. Chandran, M. Pai, E. A. Blomme, G. C. Hsieh, M. W. Decker and P. Honore, "Pharmacological Modulation of Movement-Evoked Pain in a Rat Model of Osteoarthritis,” European Journal of Pharmacology, Vol. 613, No. 1-3, 2009, pp. 39-54. doi:10.1016/j.ejphar.2009.04.009

[15] R. Combe, S. Bramwell and M. J. Field, "The Monosodium Iodoacetate Model of Osteoarthritis: A Model of Chronic Nociceptive Pain in Rats?” Neuroscience Letters, Vol. 370, No. 2-3, 2004, pp. 236-240. doi:10.1016/j.neulet.2004.08.023

[16] K. L. Chu, P. Chandran, S. K. Joshi, M. F. Jarvis, P. R. Kym and S. McGaraughty, "TRPV1-Related Modulation of Spinal Neuronal Activity and Behavior in a Rat Model of Osteoarthritic Pain,” Brain Research, Vol. 1369, 2011, pp. 158-166. doi:10.1016/j.brainres.2010.10.101

[17] E. Schött, O. G. Berge, K. Ängeby-Möller, G. Hammarström, C. J. Dalsgaard and E. Brodin, "Weight Bearing as an Objective Measure of Arthritic Pain in the Rat," Journal of Pharmacological and Toxicological Methods, Vol. 31, No. 2, 1994, pp. 79-83. doi:10.1016/1056-8719(94)90046-9

[18] B. S. Boyd, C. Puttlitz, L. J. Noble-Haeusslein, C. M. John, A. Trivedi and K. S. Topp, "Deviations in Gait Pattern in Experimental Models of Hindlimb Paresis Shown by a Novel Pressure Mapping System," Journal of Neuroscience Research, Vol. 85, No. 10, 2007, pp. 22722283. doi:10.1002/jnr.21366

[19] H. Rashid, Y. Theberge. S. J. Elmes, M. N. Perkins and F. McIntosh, "Pharmacological Validation of Early and Late Phase of Rat Monoiodoacetate Model Using the Tekscan System," European Journal of Pain, Vol. 17, No. 2, 2012, pp. 210-222. doi:10.1002/j.1532-2149.2012.00176.X

[20] J. Ferreira-Gomes, S. Adães and J. M. Castro-Lopes, “Assessment of Movement-Evoked Pain in Osteoarthritis by the Knee-Bend and Catwalk Tests: A Clinically Relevant Study," The Journal of Pain, Vol. 9, No. 10, 2008, pp. 945-954. doi:10.1016/j.jpain.2008.05.012

[21] S. S. Min, J. S. Han, Y. I. Kim, H. S. Na, Y. W. Yoon, S. K. Hong and H. C. Han, "A Novel Method for Convenient Assessment of Arthritic Pain in Voluntarily Walking Rats," Neuroscience Letters, Vol. 308, No. 2, 2001, pp. 95-98. doi:10.1016/S0304-3940(01)01983-8

[22] P. Tétreault, M. Dansereau, L. Doré-Savard, N. Beaudet and P. Sarret, "Weight Bearing Evaluation in Inflamematory, Neuropathic and Cancer Chronic Pain in Freely Moving Rats,” Physiology \& Behavior, Vol. 104, No. 3, 2011, pp. 495-502. doi:10.1016/j.physbeh.2011.05.015

[23] K. A. Clarke, S. A. Heitmeyer, A. G. Smith and Y. O. 
Taiwo, "Gait Analysis in a Rat Model of Osteoarthrosis," Physiology \& Behavior, Vol. 62, No. 5, 1997, pp. 951954. doi:10.1016/S0031-9384(97)00022-X

[24] J. Fernihough, C. Gentry, M. Malcangio, A. Fox, J. Rediske, T. Pellas, B. Kidd, S. Bevan and J. Winter, "Pain Related Behavior in Two Models of Osteoarthritis in the Rat Knee,” Pain, Vol. 112, No. 1-2, 2004, pp. 1-11. doi:10.1016/j.pain.2004.08.004

[25] A. M. Fendrick and B. P. Greenberg, "A Review of the Benefits and Risks of Nonsteroidal Anti-Inflammatory Drugs in the Management of Mild-to-Moderate Osteoarthritis," Osteopathic Medicine and Primary Care, Vol. 3, 2009, pp. 1-7. doi:10.1186/1750-4732-3-1

[26] T. W. Towheed and M. C. Hochberg, “A Systemic Review of Randomized Controlled Trials of Pharmacological Therapy in Osteoarthritis of the Knee, with an Emphasis on Trial Methodology," Seminars in Arthritis and Rheumatism, Vol. 26, No. 5, 1997, pp. 755-770. doi:10.1016/S0049-0172(97)80043-1

[27] J. Ferreira-Gomes, S. Adães, M. Mendonça and J. M.
Castro-Lopes, “Analgesic Effects of Lidocaine, Morphine and Diclofenac on Movement-Induced Nociception, as Assessed by the Knee-Bend and Catwalk Tests in a Rat Model of Osteoarthritis," Pharmacology Biochemistry and Behavior, Vol. 101, No. 4, 2012, pp. 617-624. doi:10.1016/j.pbb.2012.03.003

[28] J. R. Caldwell, R. J. Rapoport, J. C. Davis, H. L. Offenberg, H. W. Marker, S. H. Roth, W. Yuan, L. Eliot, N. Babul and P. M. Lynch, "Efficacy and Safety of a OnceDaily Morphine Formulation in Chronic, Moderate-toSevere Osteoarthritis Pain: Results from a Randomized, Placebo-Controlled, Double-Blind Trial and An open-Label Extension Trial,” Journal of Pain and Symptom Management, Vol. 23, No. 4, 2002, pp. 278-291. doi:10.1016/S0885-3924(02)00383-4

[29] I. Robinson, B. Sargent and J. P. Hatcher, "Use of Dynamic Weight Bearing as a Novel End-Point for the Assessment of Freund's Complete Adjuvant Induced Hypersensitivity in Mice," Neuroscience Letters, Vol. 524, No. 2, 2012, pp. 107-110. doi:10.1016/j.neulet.2012.07.017 